\title{
COVID-19 and the eye immunity: lesson learned from the past and possible new therapeutic insights
}

\author{
Piergiorgio Neri (i) - Francesco Pichi
}

Published online: 20 April 2020

(C) Springer Nature B.V. 2020

Corona virus represents nowadays the hot topic in the scientific world due to the outbreak of a novel serotype formerly named coronavirus disease (COVID)-19 and now identified as severe acute respiratory syndrome (SARS)-COV-2 [1].

The disease has been firstly reported in Wuhan, Yubei District of the Republic of China, when in December 2019 its outbreak affected around 80,000 people and provoked about 3000 deaths due to pulmonary complications [1]. The virus rapidly spread all over the world and was recognized as a pandemic by the World Health Organization (WHO) on March 11,2020 [2].

In 4 months, the disease was recognized in about $1,521,252$ people and made 92,798 victims mainly in elderly and compromised patients. On an official report released on April 10, 2020, SARS-COV-2 carried a mortality rate around $6.1 \%$ compared to influenza that is supposed be around $1 \%$ [3].

All countries are encountering massive and dramatic problems in terms of prevention and

P. Neri $(\bowtie) \cdot$ F. Pichi

The Eye Institute, Cleveland Clinic Abu Dhabi, Al Maryah Island, PO Box 112412, Abu Dhabi, UAE

e-mail:pg.neri@gmail.com

P. Neri · F. Pichi

Cleveland Clinic Lerner College of Medicine, Case

Western University, Cleveland, $\mathrm{OH}$, USA sustainability of treatments for this disease which does not have a universally agreed therapeutic protocol.

Current management of COVID-19 is mainly supportive, even though the medical literature has reported several rescue strategies which might minimize the mortality rate in those who have severe pulmonary complications.

Hydroxychloroquine and azithromycin combination seems to be a valid possible therapeutic protocol [4] and is currently under urgent investigation.

Unfortunately, the real challenge is represented by those cases where the severe form of SARS-COV-2 interstitial pneumonia occurs, with acute respiratory distress syndrome (ARDS) that might lead to fatal events [5].

The urgent need to find a treatment for such lifethreatening condition has led to off-label approaches based on the possible hypercytokinemia with multiorgan failure [6].

An experimental protocol using an anti-interleukin (IL)-6 receptor monoclonal antibody named tocilizumab (Actemra, Roche Pharma (Schweiz) Ltd, B2084B21) has given hope of a possible effective therapy. Tocilizumab was used as a rescue treatment in a series of patients affected by severe interstitial pneumonia by Zhang et al. [7] which observed an evident improvement in both systemic symptoms and the specific pulmonary impairment within a few days. 
These interesting results were followed by a series of other publications speculating on how the inhibition of immune checkpoints might have exerted a possible good control of the disease.

On the other hand, the severe contingent situation created by the outbreak of SARS-COV-2 infection does not allow to have good models to rely on.

In the medical literature, SARS coronavirus is associated with the occurrence of hemophagocytic lymphohistiocytosis (sHLH) which represents a lifethreatening hyperinflammatory syndrome leading to a rapid multiorgan deterioration due to severe hypercytokinemia [6].

At this stage, can the ocular immune system represent a valid model upon which to rely in order to hypothesize a possible therapeutic strategy?

In 1990, Robbins et al. reported on a possible animal model where murine coronavirus induced an acute and long-lasting disease of the retina [8]. This model was identified as experimental coronavirus retinopathy (ECOR) [8].

Few months later, the same group of researchers reported that murine coronavirus had a specific retinotropism when introduced by several direct routes [9].

The researchers found that the viral insult leads to a progressive impairment of all the structures of the retinal tissue, including photoreceptors and retinal pigment epithelium (RPE).

Murine coronavirus showed an evident tropism to the neural tissue of the retina which was independent of route of inoculation, such as onto the cornea, the anterior chamber (AC), intravitreally, intracerebrally, or intranasally even. Interestingly, viral antigens were located primarily in the inner nuclear layer, photoreceptors, Muller cells, and RPE. On the other hand, at day 10, ganglion cell layer was the only and still infected, indicating a spontaneous clearance of the virus from the other retinal tissues. This trend in affecting the nervous system might be the case of SARS-COV-2 as well, since a recent report described Guillan-Barrè syndrome as the first manifestation of the disease [10].

Interestingly, infection induces fibrosis of RPE cells as well as moderate retinal atrophy in the long term, which suggests a certain role played by fibroblasts in attempting a repair of the damaged tissue.

Although ECOR was used to study retinal degeneration specifically, it might represent a possible experimental model for interesting speculations on how to approach severe SARS-COV-2 pulmonary complications.

Going through the medical literature, very little information is available on the possible role played by the immune system in lung involvement, which shows moderate multinucleated giant cells, minimal lymphocytes, eosinophils, CD4-positive $\mathrm{T}$ cells, and neutrophils [11]. Lungs blood vessels of alveolar septum are congested, edematous, and widened, with modest infiltration of monocytes and lymphocytes. Hyaline thrombi are also found in a minority of microvessels, as well as local hemorrhage in lung tissue, organization of exudates in some alveolar cavities, and pulmonary interstitial fibrosis. On the other hand, all those findings were taken postmortem and there is lack of data on different phases of the disease.

Looking at the ECOR model, it gives the impression that coronavirus creates two different phases: the first is represented by the primary infection which induces the triggering of the immune system, while the second phase is likely to be an autoimmune disease where the role of the severe postviral inflammation represents a severe occurrence worth of prompt intervention.

Coronavirus animal model suggests that such hypothesis might be justified [9]: as we previously said, Coronavirus was detected into the ganglion cell layer only after 10 days, even though in $26 \%$ of the experimental animals a severe and massive inflammation of most ocular structures was observed. The authors speculated that gliosis might have been present in affected retinas as the result of breakdown of the blood-ocular barrier which can allow coronavirus-bearing macrophages to access the eye.

Regarding the specific vascular involvement, massive inflammation leads to damage to the walls of veins, evidenced by loss of continuity and erythrocytes extravasation into the surrounding tissue. Similarly, the lung polythrombosis observed in SARS-COV-2 cases seems to be a result of the interstitial inflammation where hyaline thrombi of the microvessels might be induced by the perivasculitis affecting the vessels walls embedded into the affected tissue [11].

At this stage, the therapeutic strategy is still opened for discussion and might offer a series of fascinating hypotheses for a rescue treatment in severe SARSCOV-2 pneumonia. 
Albeit it is true that anti-IL-6 receptor monoclonal antibody has given promising results for the control of severe SARS-COV-2 pneumonia, it is interesting to notice that retinal degeneration in ECOR is associated with an evident increase in TNF-alpha, as well as soluble TNFR2, inducing an anomaly of TNF-alpha signaling [12].

The clinical picture offered by SARS-COV-2 pneumonia seems to present a series of similarities to so-called interstitial pneumonia with autoimmune features (IPAF) recently described by the European Respiratory Society and the American Thoracic Society [13].

In IPAF, the immune reaction plays a primary role in its pathogenesis. However, there is no general agreement on any recommendations; therefore, even the diagnosis and management are left to the individual caregiver [13].

While the use of anti-TNF-alpha appears quite risky due to the possible occurrence of fulminant tuberculosis or might play a very limited role, other new generation immunosuppressives, such as intravenous immunoglobulin, selective IL-1 cytokine blockade, and JAK inhibition [6], were proposed.

However, it is unclear why the scientific world is still strongly reluctant to promptly use steroids to target the second phase of the disease, while this is not the case for monoclonals.

Similarly to Ebola Virus Disease (EVD), where steroids were successfully used even though empirically, the number of circulating $\mathrm{T}$ lymphocytes is dramatically reduced in severe cases, and elevated cytokine levels in the serum are observed [14].

Under that light, steroids might appear a potent weapon against fatal immune system activation. Since World Health Organization provided no guideline on corticosteroids use for the management of SARSCOV-2, they can be only used empirically with no indication on timing, duration, and dosage. In addition, early use of steroids seems to exert a more than promising control of inflammatory response in other severe viral diseases [14].

The same issue was addressed several times for more common diseases like herpes virus encephalitis [15], which is universally recognized as a postviral autoimmune response. Herpetic postviral central nervous system inflammation has corticosteroids as the first line drug in the therapeutic armamentarium [16] and might be considered a possible model for a prompt response in treating earlier life-threatening forms of SARS-COV-2.

On the basis of the current knowledge, corticosteroids might be a potent therapeutic weapon for the second phase of the disease, when the immune system targets dramatically multiple organs and specifically the lungs, while it has to be kept in mind that their use can be risky when the activation of inflammatory processes remains within the normal range.

However, the risk/benefit ratio of corticosteroids for severe form of SARS-COV-2 disease should be clarified by the health authorities [17], as well as the weight of cost-effectiveness compared to other therapies like monoclonals.

Moreover, the SARS-COV-2 pneumonia animal model described in 2010 by Smits et al. [18] presents many similarities with ECOR reported almost 2 decades earlier, in terms of viral clearance and timing of immune response.

Considering the course of the disease, it seems the supposed postcoronavirus immune response happens within 10 to 14 days after the onset of the disease in both models. Consequently, that might be the appropriate timing to treat, while the viral insult might be over already as per the animal model. At that time, a prompt intervention with no delay either by monoclonals or by steroids may rescue the patient and avoid the local immune-driven irreversible tissue damage.

It might have been appropriate to test these hypotheses by an appropriate trial, but nowadays time does not play in favor and there is the urgent need of a prompt and brave action.

\section{References}

1. Seah I, Agarwal R (2019) Can the coronavirus disease 2019 (COVID-19) affect the eyes? A review of coronaviruses and ocular implications in humans and animals. Ocul Immunol Inflamm 28(3):391-395

2. WHO (2020) WHO director-general's opening remarks at the media briefing on COVID-19-11 March 2020. https:// www.who.int/dg/speeches/detail/who-director-general-sopening-remarks-at-the-media-briefing-on-covid-19-11march-2020. Accessed 11 April 2020

3. WHO (2020) Coronavirus disease 2019 (COVID-19) situation report-81. https://www.who.int/docs/default-source/ coronaviruse/situation-reports/20200410-sitrep-81-covid19.pdf?sfvrsn=ca96eb84_2. Accessed 11 April 2020

4. Colson P, Rolain JM, Lagier JC et al (2020) Chloroquine and hydroxychloroquine as available weapons to fight 
COVID-19. Int J Antimicrob Agents. https://doi.org/10. 1016/j.ijantimicag.2020.105932

5. Sohrabi C, Alsafi Z, O'Neill N et al (2020) World Health Organization declares global emergency: a review of the 2019 novel coronavirus (COVID-19). Int J Surg 76:71-76

6. Mehta P, McAuley DF, Brown M et al (2020) HLH across speciality collaboration, UK COVID-19: consider cytokine storm syndromes and immunosuppression. Lancet 395(10229):1033-1034. https://doi.org/10.1016/s01406736(20)30628-0

7. Zhang C, Wu Z, Li JW et al (2020) The cytokine release syndrome (CRS) of severe COVID-19 and Interleukin-6 receptor (IL-6R) antagonist Tocilizumab may be the key to reduce the mortality. Int $\mathrm{J}$ Antimicrob Agents. https://doi. org/10.1016/j.ijantimicag.2020.105954

8. Robbins SG, Detrick B, Hooks JJ (1990) Retinopathy following intravitreal injection of mice with MHV strain JHM. Adv Exp Med Biol 276:519-524. https://doi.org/10.1007/ 978-1-4684-5823-7_72

9. Robbins SG, Detrick B, Hooks JJ (1991) Ocular tropisms of murine coronavirus (strain JHM) after inoculation by various routes. Invest Ophthalmol Vis Sci 32(6):1883-1893

10. Zhao H, Shen D, Zhou H et al (2020) Guillain-Barré syndrome associated with SARS-CoV-2 infection: causality or coincidence? Lancet Neurol [Online ahead of print]

11. Yao XH, Li TY, He ZC et al (2020) A pathological report of three COVID-19 cases by minimally invasive autopsies. Zhonghua Bing Li Xue Za Zhi 49:E009 (in Chinese)
12. Hooper LC, Chin MS, Detrick B, Hooks JJ (2005) Retinal degeneration in experimental coronavirus retinopathy (ECOR) is associated with increased TNF-alpha, soluble TNFR2 and altered TNF-alpha signaling. J Neuroimmunol 166(1-2):65-74

13. Fischer A, Antoniou KM, Brown KK et al (2015) "ERS/ ATS task force on undifferentiated forms of CTD-ILD". An official European respiratory society/American thoracic society research statement: interstitial pneumonia with autoimmune features. Eur Respir J 46(4):976-987

14. Xu J, Tan DY, Fu YY et al (2015) Do corticosteroids have a role in treating Ebola virus disease? Sci China Life Sci 58(1):111-113

15. Shang L, Zhao J, Hu Y et al (2020) On the use of corticosteroids for 2019-nCoV pneumonia. Lancet 395(10225):683-684

16. Stahl JP, Mailles A (2019) Herpes simplex virus encephalitis update. Curr Opin Infect Dis 32(3):239-243

17. Chaudhuri A, Kennedy PGE (2002) Diagnosis and treatment of viral encephalitis. Postgrad Med J 78:575-583

18. Smits SL, de Lang A, van den Brand JMA et al (2010) Exacerbated innate host response to SARS-CoV in aged non-human primates. PLoS Pathog 6(2):e1000756

Publisher's Note Springer Nature remains neutral with regard to jurisdictional claims in published maps and institutional affiliations. 